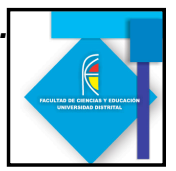

\title{
HABILIDADES DE PENSAMIENTO CIENTÍFICO: UNA ESTRATEGIA DIDÁCTICA BASADA EN TRABAJOS PRÁCTICOS
}

\section{SCIENTIFIC THINKING SKILLS: TEACHING STRATEGY BASED ON PRACTICAL WORK}

\author{
Martha Andrea Vargas Guerrero ${ }^{1}$ \\ María Cristina Cifuentes Arcila
}

\begin{abstract}
Resumen.
Esta investigación se orienta a través de la pregunta: ¿Cómo contribuye una estrategia didáctica en el Desarrollo de las Habilidades de Pensamiento Científico de los niños y niñas de sexto grado que asisten al Colegio Hacienda Los Alcaparros? Dos elementos permiten justificar esta investigación: la necesidad de pensar la práctica pedagógica como un espacio de reflexión y producción de conocimiento educativo; y la necesidad de generar didácticas que se ajusten a las singularidades de las realidades escolares. Los 22 participantes de esta investigación tienen edades entre los 11 y los 13 años de edad y pertenecen a los estratos socio-económicos 5 y 6 de la ciudad de Bogotá.. El enfoque de la investigación es cualitativo-interpretativo apoyado en los planteamientos de Erickson (1989).Los resultados indican que la estrategia didáctica funcionó en la medida en que posibilitó el Desarrollo de Habilidades de Pensamiento Científico en los estudiantes, las habilidades para la gestión y evaluación de la evidencia, así como la construcción y justificación de conclusiones para una audiencia se desarrolló de manera diferencial en cada uno de los casos estudiados.
\end{abstract}

Palabras Clave: Habilidades de pensamiento científico, Estrategia didáctica, Gestión y evaluación de la evidencia, Construcción y justificación de conclusiones.

\section{Abstract.}

This research present a qualitative is guided by the question: How does a teaching strategy in the Development of Scientific Thinking Skills children attending sixth grade at

\footnotetext{
${ }^{1}$ Universidad Pedagógica Nacional

${ }^{2}$ Universidad Pedagógica Nacional
} 


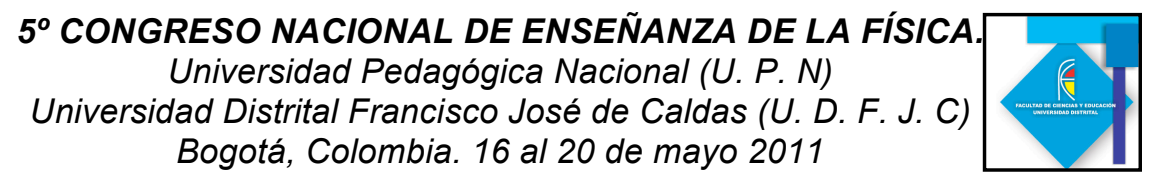

Colegio Hacienda Los Alcaparros? Two factors justify this research: the need to consider pedagogic practice as a space for reflection and production of educational knowledge and the need to generate teaching to fit the peculiarities of educational situations. The 22 participants in this study are aged between 11 and 13 years old and belong to the socioeconomic strata 5 and 6 of the city of Bogotá. The research approach is qualitative, interpretive approaches supported by Erickson (1989). The results indicate that the teaching strategy worked to the extent that enabled the development of Scientific Thinking Skills in students the skills to manage and evaluation of evidence and the construction and justification of conclusions for a hearing was held differentially in each of the cases studied.

Keywords: Scientific thinking skills, teaching strategies, management and evaluation of evidence, construction and justification of conclusions.

\section{Presentación}

Este trabajo surgió del reconocimiento de la importancia de pensar la enseñanza como un proceso de reflexión y producción de conocimiento sobre la educación en ciencias, en el contexto de la práctica pedagógica de una profesora principiante en un colegio de educación básica secundaria. En este contexto surgió la necesidad de desarrollar una estrategia didáctica que ofreciera a los estudiantes ricas y variadas oportunidades para el desarrollo de sus habilidades de pensamiento científico, en las clases de ciencias.

Varios elementos fueron contemplados para el desarrollo de dicha didáctica, tales como: estructurar la didáctica alrededor de seis trabajos prácticos y la física del movimiento de los cuerpo, y orientar la didáctica para propiciar el desarrollo de las habilidades necesarias para el análisis e interpretación de datos, y la obtención y justificación de conclusiones dentro de una actividad de investigación. La decisión de desarrollar una didáctica alrededor de trabajos prácticos se apoyó en el reconocimiento de que dichos trabajos permiten a los estudiantes experimentar las cosas directamente y manejar los objetos y los organismos por sí mismos, para así desarrollar un bagaje de experiencia personal; y desarrollar e intensificar sus conocimientos conceptuales a través de la exploración, elaboración y supervisión de sus ideas, y la comparación con las aportadas por la experiencia (Hodson, 1993). La decisión de estructurar la didáctica sobre la física del movimiento reconoce que las habilidades de pensamiento científico son inseparables de los contenidos disciplinares. Finalmente, el interés por las habilidades de análisis e interpretación de datos, y la obtención y justificación de conclusiones, reconoce la importancia que tiene los procesos de dar sentido a los datos y de construir un argumento soportado en dichos datos, en los procesos de construcción de conocimiento de los estudiantes (Edelson \& Reiser, 2006).

Es importante destacar que el eje central de la estrategia didáctica fue el uso de los trabajos prácticos como estrategia de enseñanza-aprendizaje, sin que por ello no se recurriera a otras estrategias de enseñanza complementarias tales como: explicaciones magistrales por parte del profesor, resolución de problemas de lápiz y papel, y lecturas complementarias de su texto guía. Por lo tanto, del total de las clases organizadas para 


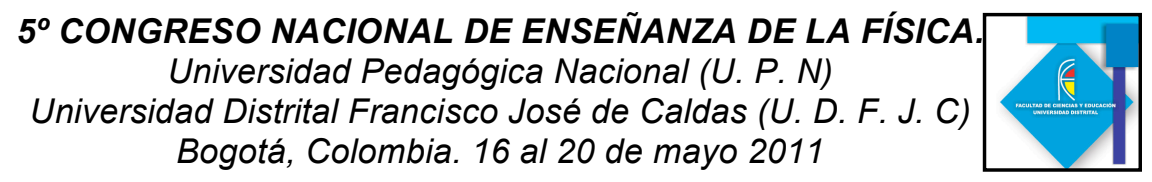

este grupo de estudiantes, solamente el $50 \%$ estuvieron destinadas al desarrollo de trabajos prácticos (TP).

La estrategia didáctica se desarrolló a lo largo de seis semanas de clase, en cada semana, las clases se organizaron de la siguiente manera: Las clases de los días martes y miércoles - a cargo del profesor titular de ciencias- se orientaron a la discusión de las ideas previas de los estudiantes sobre los fenómenos físicos objeto de estudio, y de los conceptos científicos referidos a dichos fenómenos, con el objeto de elaborar un lenguaje común; la clase de los días lunes - a cargo de la profesora practicante- se destinaron al desarrollo de trabajos prácticos, con el fin de que los estudiantes a través de la exploración, elaboración y supervisión de sus ideas, y la comparación de dichas ideas con las aportadas por las experiencias construyeran su conocimiento sobre la disciplina y simultáneamente desarrollaran sus habilidades de pensamiento científico, específicamente las habilidades de gestión y evaluación de la evidencia; y, finalmente, las clase de los días viernes -también a cargo de la profesora practicante- se destinaron a la construcción de conclusiones, por parte de los estudiantes, a partir de la evidencia colectada en sus trabajos prácticos, y la construcción de argumentos de justificación de dichas conclusiones para compartir con sus compañeros de clase.

Los contenidos cubiertos por las actividades experimentales fueron: trayectoria, desplazamiento, rapidez, velocidad, aceleración, las leyes de Newton, la fricción, y la gravedad. Cada uno de los trabajos prácticos presentados en la estrategia de enseñanza tiene unos objetivos tanto disciplinares como orientados al desarrollo de habilidades de pensamiento científico. En cuanto a los objetivos que guiaron el desarrollo de habilidades de pensamiento científico fueron iguales en cada uno de los contenidos, con ello se pretendió que independientemente del tema a tratar el estudiante estuviera en capacidad de: a) identificar variables y vincular su influencia en diferentes fenómenos para lograr una capacidad de síntesis, b) registrar las observaciones y resultados utilizando esquemas, gráficos y tablas, c) utilizar las matemáticas como una herramienta para organizar, analizar y presentar datos, d) utilizar como estrategia para organizar la información tablas de datos numéricos, e) transformar tablas de datos en gráficas de dos dimensiones $\mathrm{f}$ ) sacar conclusiones a partir de los datos recolectados y organizados.

Pero más allá de desarrollar una didáctica, el propósito central de esta investigación fue describir el desarrollo de las habilidades de pensamientos de los estudiantes, en el marco de una didáctica intencionalmente diseñada para ellos. De allí que la metodología y conclusiones, que se presentan a continuación, se refieren a dichas habilidades.

\section{Metodología.}

Los participantes de este estudio fueron 22 estudiantes, 5 niñas y 17 niños con edades entre los 11 y los 13 años, provenientes de los estratos socio-económicos 5 y 6 , que asistían a la institución donde la profesora principiante desarrollo sus prácticas docentes.

Las fuentes primarias de datos utilizadas en este estudio fueron: (a) las grabaciones de audio de cada una de las clases (once clases en total), que fueron trascritas, y (b) una entrevista semi-estructurada que se realizó a un grupo focal al finalizar la implementación 


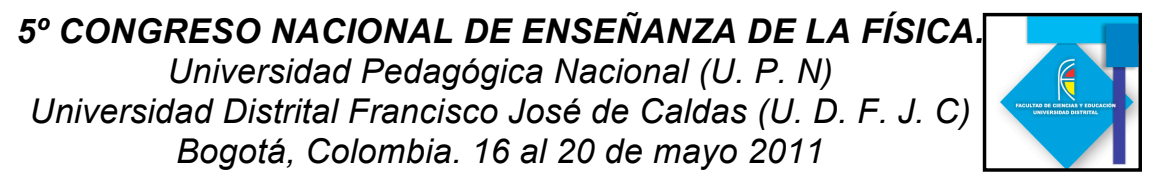

de la estrategia didáctica para recoger algunas impresiones y que constituyó un ejercicio metacognitivo. Para el presente trabajo no se utilizan fuentes secundarias de datos.

Para el análisis de la información recolectada, se implementaron las estrategias interpretativas de análisis de datos propuestas por Erickson (1986/1989). Cada una de las clases al igual que la entrevista final, fueron grabadas y posteriormente trascritas. Se crearon una serie de valoraciones y códigos. Para su posterior análisis fueron agrupadas de acuerdo con sus respectivos grupos de trabajo y procesadas mediante el programa para computador AtlasTi, herramienta informática que facilita el análisis de información cualitativa.

Para la correcta organización, análisis y evaluación de la información recolectada, se construyó una matriz de valoración, que da cuenta de cómo los participantes gestionan y evalúan la evidencia, y de cómo construyen una serie de argumentaciones para una audiencia. En general, se realizaron cinco categorías con sus respectivos códigos. Estas categorías se organizaron de la siguiente manera: (1) Registro -sobre cómo los estudiantes registran sus mediciones, la utilización de instrumentos, los patrones de medición que ellos utilizan, así como la precisión y exactitud en la medida, (Zimmerman, 2007) (2) Representación de la evidencia - sobre la clase de representaciones que realizan los estudiantes, en este sentido, pueden ser, diagramas simples, diagramas sintéticos, diagramas analíticos, gráficas, mapas, tablas o líneas de tiempo (Coleman, McTigue, Smolkin, 2010; Waldrip, Prain, Carolan \& Jim, 2010) (3) Inferencia/Conclusión - en relación a la clase de inferencias que construían los participantes, que pueden ser, precausales, causales, no causales, indeterminadas o inclusiones falsas (Piaget, 1972; Zimmerman, 2007), (4) Producción de justificaciones - en relación con la clase de justificaciones que los participantes construían, estas justificaciones pueden ser clasificadas según su fuente y según su calidad, (Kuhn \& Udell, 2010; Henao \& Stipcich, 2008) y (5) Producción de explicaciones - sobre cómo los participantes realizaban sus explicaciones, estas pueden ser clasificadas como, prácticas, descriptivas e interpretativas.

La configuración de los casos de estudio, como grupos, se realizó teniendo en cuenta algunos elementos como: (a) la cantidad de sesiones en la que los mismos participantes decidieron trabajar juntos y la frecuencia con la que permanecieron juntos a lo largo de todas las clases, y (b) los participantes que no mostraron ninguna frecuencia al trabajar con el mismo grupo de trabajo, sino que a lo largo de la implementación de la estrategia didáctica realizaron su trabajo con diferentes grupos. Estos últimos no son objeto de investigación en el presente estudio. Así pues, para el análisis se construyen cuatro grupos que se organizan de la siguiente manera.

\section{Conclusiones}

La pregunta de investigación que guió este estudio, estaba orientada a saber cómo contribuye una estrategia didáctica en el desarrollo de Habilidades de Pensamiento Científico de los niños y niñas de sexto grado que asisten al Colegio Hacienda los Alcaparros. Pues bien, tras el análisis realizado se encuentra que el Desarrollo de Habilidades de Pensamiento Científico en niños se da de manera diferencial en cada uno de ellos. Las habilidades desarrolladas en relación con la gestión y evaluación de la 


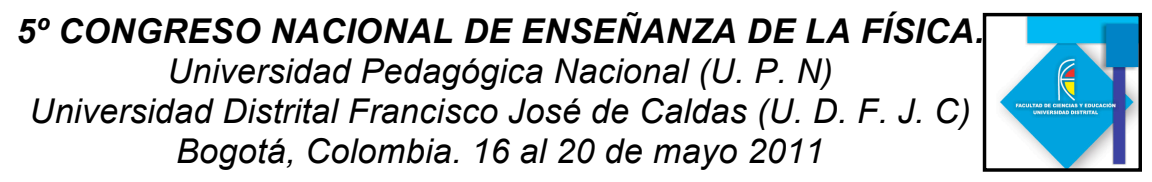

evidencia, así como para la construcción y justificación de conclusiones para una audiencia, se desarrolla de manera satisfactoria en solo dos de los grupos estudiados.

Algunos resultados sobresalientes en esta investigación, en relación con la habilidad para gestionar la evidencia, muestran que muchas veces ante la falta de instrumentos adecuados los estudiantes crean patrones de medida. Así mismo, es notorio el desarrollo en relación con la identificación de variables (que en principio no se les pide medir), pero que a consideración de ellos es importante para la mejor comprensión del fenómeno

estudiado. En relación con las representaciones de los estudiantes, es posible evidenciar que utilizan diagramas simples, gráficas y tablas, como herramientas para organizar la información recopilada en los procesos de experimentación. Dejando de lado, representaciones como los diagramas sintéticos, diagramas analíticos, mapas y líneas de tiempo. Las tablas siempre fueron la herramienta favorita para presentar la evidencia y la calidad de las mismas mejoró dado el hecho de que en algunas actividades se requería unas tablas más complejas en comparación con las utilizadas en las primeras clases.

En relación con la habilidad para evaluar la evidencia, los estudiantes utilizan razonamientos muy variados (precausal, causal, no causal, indeterminado, inclusión falsa) aunque es posible identificar diferenciación entre estos y los contextos en los cuales son usados. Por ejemplo, las inferencias precausales son usadas a lo largo de la implementación de toda la estrategia didáctica, pero estas son usadas de diferentes formas, al iniciar las que cobran mayor fuerza son las expresiones como "porque si", "Debe ser así", etc., la atribución de animismo a los objetos no es abandonada pero esta es usada para tratar de explicar conceptos físicos.

En relación con la construcción y justificación de conclusiones para una audiencia, los estudiantes realizan justificaciones basados tanto en la teoría como en la evidencia recogida en los procesos de experimentación, sin embargo, algunas veces no es posible identificar claramente en cuál de estas dos fuentes los estudiantes basan sus justificaciones, debido esto a la fuerte implicación de sus creencias en las justificaciones realizadas por ellos. Esto corresponde claramente con los trabajos reseñados en la revisión de Zimermman, más específicamente con la relación dialógica existente entre teoría y evidencia que establece que la forma en que los niños y niñas evalúan la evidencia para hacer las inferencias que sustentan una conclusión está relacionada con sus creencias en relación a un fenómeno explorado y con sus conocimientos de las teorías científicas.

En términos generales, podemos establecer que la estrategia didáctica funcionó en la medida en que posibilitó el Desarrollo de Habilidades de Pensamiento Científico en los estudiantes, adicionalmente fue de nuestro interés evaluar no solo el Desarrollo de Habilidades de Pensamiento Científico, sino también la estrategia didáctica y el propio desempeño profesional. La estrategia didáctica mostró una de las formas de enseñar física en el aula de una manera diferente, donde los estudiantes no solo señalaron divertirse aprendiendo, sino interactuar y aprender a través de la argumentación de sus conclusiones. Para evaluar el propio desempeño profesional, podemos establecer que la realización de este estudio nos ha enseñado algunas lecciones de nuestra propia práctica, y el reconocimiento de algunas debilidades como la falta y búsqueda de estrategias 


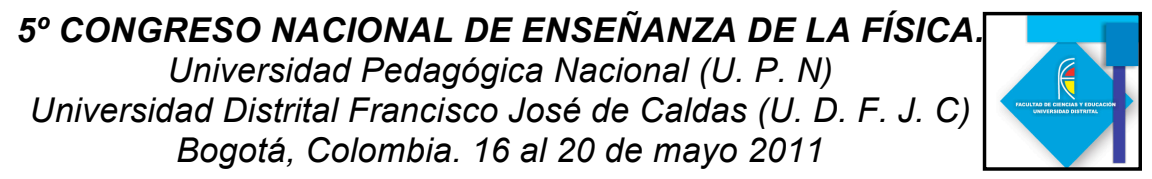

pedagógicas para la gestión del aula. A pesar de ello fue una excelente primera oportunidad para desempeñarme como futura maestra y plantear desde mi experiencia una serie de posibilidades en la enseñanza de la física.

\section{Referencias Bibliográficas}

Beaumont-Walters, Y., Soyibo, K., (2001) An analysis of high school students' performance on five integrated science process skills, Research in Science \& Technological Education,19(2),133-145.

Coleman, J., McTigue, E., Smolkin, L., (2010) Elementary Teachers' Use of Graphical Representations in Science Teaching, Journal of Science Teacher Education, 2010(1), 131.

Edelson, D. C., Reiser, B. J., (2006) Making Authentic Practices Accessible to Learners En: R. K. Sawyer, (Eds), The Cambridge Handbook of the Learning Sciences. (pp $335-354)$ New York: R. Keith Sawyer.

Erickson, F. (1989). Métodos cualitativos de investigación sobre la enseñanza. En M. Wittrock (comp.), La investigación de la enseñanza, II: métodos cualitativos y de observación (G. Vitale, Trad., pp. 195 - 301). Barcelona: Ed. Paidós (Trabajo original publicado en 1986).

Henao, B., Stipcich, M., (2008) Educación en ciencias y argumentación: la perspectiva de Toulmin como posible respuesta a las demandas y desafíos contemporáneos para la enseñanza de las Ciencias Experimentales. Revista Electrónica de Enseñanza de las ciencias, 7(1). Consultado el 11 de agosto de 2010 en: http://reec.uvigo.es/volumenes/volumen7/ART3_Vol7_N1.pdf

Hodson, D., (1993, septiembre) Hacia un enfoque más crítico del trabajo de laboratorio. Documento presentado al IV Congreso Internacional sobre Investigación en la Didáctica de las Ciencias y las Matemáticas, Barcelona, España.

Kuhn, D., Udell, W., (2010) The development of argument skills, Child Development, 74(5), $1245-1260$.

Ministerio de Educación Nacional. (2004). Estándares Básicos de Competencias en Ciencias Naturales y Ciencias Sociales, formar en ciencias: iel desafío! Lo que necesitamos saber y saber hacer.

Piaget, J. (1972). El Lenguaje y el Pensamiento en el Niño. (M. Riani, Trad.) Buenos Aires: Guadalupe (Trabajo original publicado 1923).

Waldrip, B., Prain, V., Carolan., Jim., (2010) Using-Modal representations to improve learning in Junior Secondary Science, Research in Science Education, 40(1), 65-80.

Zimmerman, C., (2007). The development of scientific thinking skills in elementary and middle school. Developmental Review, 27, 172 - 223. 